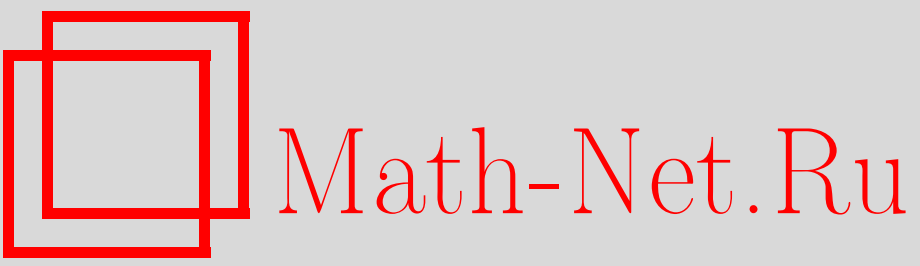

В. П. Маслов, О зависимости критерия сверхтекучести от радиуса капилляра, TMФ, 2005, том 143, номер 3, 307-327

DOI: https://doi.org/10.4213/tmf1816

Использование Общероссийского математического портала Math-Net.Ru подразумевает, что вы прочитали и согласны с пользовательским соглашением

http://www . mathnet.ru/rus/agreement

Параметры загрузки:

IP : 3.82 .47 .9

26 апреля 2023 г., 13:50:07 
ТЕОРЕТИЧЕСКАЯ

И МАТЕМАТИЧЕСКАЯ

ФИЗИКА

Том 143, № 3

июнь, 2005

(C) 2005 г.

В. П. Маслов*

\section{О ЗАВИСИМОСТИ КРИТЕРИЯ СВЕРХТЕКУЧЕСТИ ОТ РАДИУСА КАПИЛЛЯРА}

Краевые условия на границе капилляра заменены периодическими условиями Борна-Кармана, т.е. рассматривается в поперечнике двумерный тор радиуса $L_{2}$. Если скорость сверхтекучей жидкости превышает величину $8 \pi \hbar /\left(m L_{2}\right)$, то она тормозится благодаря трению о вихрь, образованный па́рами (подобными па́рам в андреевском отражении).

Ключевые слова: сверхтекучесть, ультравторичное квантование, термодинамический предел, спектр квазичастиц, толщина капилляра, вихрь.

В данной работе исходя из истинного символа ультравторично-квантованного оператора [1] получены результаты для системы большого числа тождественных бозонов в термодинамическом пределе. При этом оказывается, что термодинамический предел для уравнений характеристик, отвечающих истинному символу, является квазиклассическим пределом для этих уравнений.

Вначале напомним основные понятия ультравторичного квантования и истинного символа. Ультравторичное квантование было введено в [2], где содержится подробное обсуждение. В данной статье используется частный случай ультравторичного квантования - квантование по парам. Кроме того, далее предполагается, что частицы рассматриваемой физической системы находятся на трехмерном торе $L_{1} \times L_{2} \times L_{3}$, который далее обозначается $\mathbf{T}, L_{1}, L_{2}$ и $L_{3}$ - длины сторон этого тора. В случае квантования по парам пространством ультравторичного квантования является бозонное пространство Фока $\mathcal{F}[3]$. Это пространство состоит из векторов $\Phi$ вида

$$
\begin{aligned}
\Phi= & \sum_{M=0}^{\infty} \frac{1}{\sqrt{M}} \int \ldots \int d x_{1} \ldots d x_{2 M} \Phi_{M}\left(x_{1}, x_{2} ; \ldots ; x_{2 M-1}, x_{2 M}\right) \times \\
& \times \widehat{B}^{+}\left(x_{1}, x_{2}\right) \ldots \widehat{B}^{+}\left(x_{2 M-1}, x_{2 M}\right) \Phi_{0}
\end{aligned}
$$

где $\widehat{B}^{+}\left(x, x^{\prime}\right)$ и $\widehat{B}^{-}\left(x, x^{\prime}\right)$ - операторы рождения и уничтожения пары частищ в пространстве $\mathcal{F}, \Phi_{0}$ - вакуумный вектор в пространстве $\mathcal{F}$, переменные $x_{i}, i=1,2, \ldots$, принимают значения на торе $\mathbf{T}$, а функции $\Phi_{M}\left(x_{1}, x_{2} ; \ldots ; x_{2 M-1}, x_{2 M}\right) \in L_{2}\left(\mathbf{T}^{2 M}\right), M=$

\footnotetext{
* Московский государственный университет, Москва, Россия. E-mail: v.p.maslov@mail.ru
} 
$0,1, \ldots$, симметричны относительно перестановок любых пар переменных $\left(x_{2 j-1}, x_{2 j}\right)$ и $\left(x_{2 i-1}, x_{2 i}\right)$ и удовлетворяют условию

$$
\sum_{M=0}^{\infty} \int \ldots \int d x_{1} \ldots d x_{2 M}\left|\Phi_{M}\left(x_{1}, x_{2} ; \ldots ; x_{2 M-1}, x_{2 M}\right)\right|^{2}<\infty .
$$

Операторы рождения и уничтожения удовлетворяют стандартным бозонным коммутационным соотношениям [3]:

$$
\begin{aligned}
{\left[\widehat{B}^{-}\left(x_{1}, x_{2}\right) \widehat{B}^{+}\left(x_{1}^{\prime}, x_{2}^{\prime}\right)\right] } & =\delta\left(x_{1}-x_{1}^{\prime}\right) \delta\left(x_{2}-x_{2}^{\prime}\right), \\
{\left[\widehat{B}^{ \pm}\left(x_{1}, x_{2}\right), \widehat{B}^{ \pm}\left(x_{1}^{\prime}, x_{2}^{\prime}\right)\right] } & =0,
\end{aligned}
$$

где $\delta(x-y)$ - дельта-функция Дирака на торе $\mathbf{T}$, а вакуумный вектор обладает следующим свойством:

$$
\widehat{B}^{-}\left(x_{1}, x_{2}\right) \Phi_{0}=0 \quad \forall x_{1}, x_{2} \in \mathbf{T} .
$$

При рассмотрении бозонных задач используются подпространства $\mathcal{F}_{M}^{\mathrm{Symm}}$ пространства $\mathcal{F}$, зависящие от параметра $M=0,1, \ldots$ и состоящие из таких векторов $\Phi(1)$, у которых функции $\Phi_{M^{\prime}}=0$ при $M^{\prime} \neq M$, а функция $\Phi_{M}\left(x_{1}, x_{2} ; \ldots ; x_{2 M-1}, x_{2 M}\right)$ является симметричной функцией переменных $x_{1}, x_{2}, \ldots, x_{2}$. Кроме этих подпространств используется также подпространство $\mathcal{F}^{\text {Symm }}=\bigoplus_{M=0}^{\infty} \mathcal{F}_{M}^{\text {Symm }}$. Оператор ортогонального проектирования на подпространство $\mathcal{F}^{\mathrm{Symm}}$ имеет вид

$$
\begin{aligned}
\overline{\widehat{E}} & =\sum_{M=0}^{\infty} \frac{1}{M !} \int \ldots \int d x_{1} \ldots d x_{2 M} \widehat{B}^{2}\left(x_{1}, x_{2}\right) \ldots \widehat{B}^{2}\left(x_{2 M-1}, x_{2 M}\right) \times \\
& \times \underset{x_{1} \ldots x_{2} M}{\operatorname{Symm}}\left(\widehat{B}^{-}\left(x_{1}, x_{2}\right) \ldots \widehat{B}^{-}\left(x_{2 M-1}, x_{2 M}\right)\right) \exp \left(-\iint d y d z \widehat{B}^{+}(y, z) \widehat{B}^{-}(y, z)\right),
\end{aligned}
$$

где числа над операторами обозначают порядок их действия [4], a Symm $x_{1} \ldots x_{2}$ - оператор симметризации по переменным $x_{1}, \ldots, x_{2}$. Оператор (5) также называется ультравторично-квантованным единичным оператором.

Пусть оператор Гамильтона системы $N$ бозонов на торе $\mathbf{T}$ имеет вид

$$
\widehat{H}_{N}=\sum_{j=1}^{N}\left(-\frac{\hbar^{2}}{2 m} \Delta_{j}+U\left(x_{j}\right)\right)+\varepsilon \sum_{j=1}^{N} \sum_{l=j+1}^{N} V\left(x_{j}-x_{l}\right),
$$

где $\Delta_{j}$ - оператор Лапласа, действующий на переменную $x_{j}$, а $U(x)$ и $V(x)=V(-x)-$ функции на торе $\mathbf{T}$ : соответственно потенциал внешнего поля и потенциал парного взаимодействия, $\hbar$ - постоянная Планка, $m$ - масса частиц, $\varepsilon$ - действительный параметр. Согласно [2] оператору (6) соответствует ультравторичный оператор Гамильтона вида

$$
\begin{aligned}
\widehat{\widehat{H}}= & \sum_{M=0}^{\infty} \frac{1}{M !} \int \ldots \int d x_{1} \ldots d x_{2 M} \widehat{B}^{2}\left(x_{1}, x_{2}\right) \ldots \widehat{B}^{2}\left(x_{2 M-1}, x_{2 M}\right) \times \\
& \times \widehat{H}_{2 M}\left(\underset{x_{1} \ldots x_{2 M}}{\operatorname{Symm}}\left(\widehat{B}^{-}\left(x_{1}, x_{2}\right) \ldots \widehat{B}^{-}\left(x_{2 M-1}, x_{2 M}\right)\right)\right) \times \\
& \times \exp \left(-\iint d y d z \widehat{B}^{2}(y, z) \widehat{B}^{-}(y, z)\right) .
\end{aligned}
$$


Помимо ультравторично-квантованных единичного оператора (5) и оператора Гамильтона (7) также вводится ультравторично-квантованный оператор числа частиц

$$
\begin{aligned}
\overline{\widehat{N}} & =\sum_{M=0}^{\infty} \frac{2 M}{M !} \int \ldots \int d x_{1} \ldots d x_{2 M} \widehat{\widehat{B}}^{+}\left(x_{1}, x_{2}\right) \ldots \widehat{B}^{2}\left(x_{2 M-1}, x_{2 M}\right) \times \\
& \times \underset{x_{1} \ldots x_{2 M}}{\operatorname{Symm}}\left(\widehat{B}^{-}\left(x_{1}, x_{2}\right) \ldots \widehat{B}^{-}\left(x_{2 M-1}, x_{2 M}\right)\right) \exp \left(-\iint d y d z \widehat{B}^{+}(y, z) \widehat{B}^{-}(y, z)\right) .
\end{aligned}
$$

Этот оператор обладает следуюшим свойством:

$$
\widehat{\widehat{N}} \Phi=2 M \Phi \quad \forall \Phi \in \mathcal{F}_{M}^{\mathrm{Symm}},
$$

т.е. подпространство $\mathcal{F}_{M}^{\text {Symm }}$ состоит из $2 M$-частичных векторов.

Важный результат состоит в том, что проекция ультравторично-квантованного гамильтониана (7) на подпространство $\mathcal{F}_{M}^{\text {Symm }}$ совпадает с $2 M$-частичным оператором (6). Эта теорема доказана в [2], в случае ультравторичного квантования по парам она означает следующее. Если вектор $\Phi \in \mathcal{F}_{M}^{\text {Symm }}$ имеет вид

$$
\begin{aligned}
\Phi= & \int \ldots \int d x_{1} \ldots d x_{2 M} \Psi\left(x_{1}, x_{2}, \ldots, x_{2 M-1}, x_{2 M}\right) \times \\
& \times \widehat{B}^{+}\left(x_{1}, x_{2}\right) \ldots \widehat{B}^{+}\left(x_{2 M-1}, x_{2 M}\right) \Phi_{0},
\end{aligned}
$$

где $\Psi\left(x_{1}, \ldots, x_{2 M}\right)$ - симметричная функция, то для оператора (7) справедливо равенство

$$
\begin{aligned}
\widehat{\widehat{H}} \Phi= & \int \ldots \int d x_{1} \ldots d x_{2 M} \widetilde{\Psi}\left(x_{1}, x_{2}, \ldots, x_{2 M-1}, x_{2 M}\right) \times \\
& \times \widehat{B}^{+}\left(x_{1}, x_{2}\right) \ldots \widehat{B}^{+}\left(x_{2 M-1}, x_{2 M}\right) \Phi_{0},
\end{aligned}
$$

где

$$
\widetilde{\Psi}\left(x_{1}, x_{2}, \ldots, x_{2 M-1}, x_{2 M}\right)=\widehat{H}_{2 M} \Psi\left(x_{1}, x_{2}, \ldots, x_{2 M-1}, x_{2 M}\right) .
$$

Равенства (11), (12) доказываются непосредственно действием оператора (7) на вектор (11), при этом следует учесть свойства $(3),(4)$ операторов рождения и уничтожения $\widehat{B}^{ \pm}(x, y)$ и вакуумного вектора $\Phi_{0}$, а также тот факт [3], что оператор

$$
\widehat{P}_{0}=\exp \left(-\iint d y d z \widehat{B}^{2}(y, z) \widehat{B}^{-}(y, z)\right)
$$

в пространстве $\mathcal{F}$ является оператором ортогонального проектирования на вакуумный вектор $\Phi_{0}$ и удовлетворяет равенствам

$$
\widehat{B}^{-}(x, y) \widehat{P}_{0}=0, \quad \widehat{P}_{0} \widehat{B}^{+}(x, y)=0 \quad \forall x, y \in \mathbf{T} .
$$


Понятие истинного символа было введено в статье [1]. В рассматриваемом случае ультравторичного квантования по парам истинный символ ультравторично-квантованного оператора Гамильтона (7) определяется следуюшим образом. Из (3), (14) следует, что для оператора (7) справедливо тождество

$$
\overline{\widehat{H}}=\overline{\widehat{E}} \widehat{A},
$$

где $\widehat{A}$ - следующий оператор в пространстве $\mathcal{F}$ :

$$
\begin{aligned}
\widehat{A}= & \iint d x d y \widehat{B}^{+}(x, y)\left(-\frac{\hbar^{2}}{2 m}\left(\Delta_{x}+\Delta_{y}\right)+U(x)+U(y)+\varepsilon V(x-y)\right) \widehat{B}^{-}(x, y)+ \\
& +\varepsilon \iiint \int d x d y d z d w V(x-y) \widehat{B}^{+}(x, y) \widehat{B}^{+}(z, w) \times \\
& \times\left(\widehat{B}^{-}(x, z) \widehat{B}^{-}(y, w)+\widehat{B}^{-}(x, w) \widehat{B}^{-}(y, z)\right)
\end{aligned}
$$

Истинным символом ультравторично-квантованного гамильтониана (7) является символ оператора (16). Этот символ равен пределу при $N \rightarrow \infty$ выражения, которое получается заменой в $(16)$ операторов $\widehat{B}^{+}(x, y)$ и $\widehat{B}^{-}(x, y)$ соответственно на $\phi^{+}(x, y) / \sqrt{N}$ и $\phi(x, y) / \sqrt{N}$ и умножением полученного выражения на $N$, где $N$ - число частиц, а $\phi^{+}(x, y)$ и $\phi(x, y)$ - функции на $\mathbf{T}^{2}$. Если параметр взаимодействия $\varepsilon$ равен $1 / N$, то символом оператора (16) является следующий функционал, зависящий от функций $\phi^{+}(x, y)$ и $\phi(x, y)$ :

$$
\begin{aligned}
& \mathcal{H}\left[\phi^{+}(\cdot), \phi(\cdot)\right]=\iint d x d y \phi^{+}(x, y)\left(-\frac{\hbar^{2}}{2 m}\left(\Delta_{x}+\Delta_{y}\right)+U(x)+U(y)\right) \phi(x, y)+ \\
& +\iiint \int d x d y d z d w V(x-y) \phi^{+}(x, y) \phi^{+}(z, w)(\phi(x, z) \phi(y, w)+\phi(x, w) \phi(y, z)) .
\end{aligned}
$$

Легко убедиться, что для оператора (8) имеет место аналогичное (15) тождество

$$
\overline{\widehat{N}}=\widehat{N} \overline{\widehat{E}}=\widehat{\widehat{E}} \widehat{N}
$$

где $\widehat{N}$ - оператор числа частищ в пространстве $\mathcal{F}$, имеющий вид

$$
\widehat{N}=2 \iint d x d y \widehat{B}^{+}(x, y) \widehat{B}^{-}(x, y) .
$$

Операторы (16) и (19) коммутируют между собой, и если число частиц в системе фиксировано и равно $N$, то из (19) следует, что для функций $\phi^{+}(x, y)$ и $\phi(x, y)$ выполняется условие

$$
\iint d x d y \phi^{+}(x, y) \phi(x, y)=\frac{1}{2} .
$$

В силу теоремы о совпадении ультравторично-квантованного оператора Гамильтона $(7)$ с $2 M$-частичным оператором (6) на подпространстве $\mathcal{F}_{M}^{\text {Symm }}$ из тождества (15) следует, что если вектор $\Phi$ вида (1) удовлетворяет уравнению

$$
\widehat{A} \Phi=\lambda \Phi
$$


и дополнительным условиям

$$
\widehat{N} \Phi=N \Phi, \quad \widehat{\widehat{E}} \Phi \neq 0
$$

где $N$ - четное число, то функция $\Psi\left(x_{1}, \ldots, x_{N}\right)=\operatorname{Symm}_{x_{1} \ldots x_{N}} \Phi\left(x_{1}, x_{2} ; \ldots ; x_{N-1}, x_{N}\right)$ является симметричной собственной функцией $N$-частичного оператора Гамильтона (6), а соответствуюшее собственное значение равно $\lambda$.

В случае, когда параметр взаимодействия $\varepsilon$ равен $1 / N$, асимптотика решений уравнения (21) в пределе при $N \rightarrow \infty$ определяется истинным символом (17). Уравнение (21) является частным случаем вторично-квантованных уравнений. Обший метод построения асимптотики решений вторично-квантованных уравнений, в которых при старших степенях операторов рождения и уничтожения стоит малый параметр, изложен в монографии [5]. В частности, согласно [5], если функции $\phi^{+}(x, y)$ и $\phi(x, y)$ удовлетворяют условию $(20)$ и являются решениями системы уравнений

$$
\frac{\delta \mathcal{H}}{\delta \phi^{+}(x, y)}\left[\phi^{+}(\cdot), \phi(\cdot)\right]=\Omega \phi(x, y), \quad \frac{\delta \mathcal{H}}{\delta \phi(x, y)}\left[\phi^{+}(\cdot), \phi(\cdot)\right]=\Omega \phi^{+}(x, y),
$$

где в левых частях стоят вариационные производные функционала (17), то каждому такому решению соответствует асимптотическая серия решений уравнения (21). Уравнения (23) являются аналогом уравнения Хартри, которое определяет асимптотику решений в случае обычного вторичного квантования. Уравнение Хартри, соответствуюшее оператору (6), имеет вид

$$
\left(-\frac{\hbar^{2}}{2 m} \Delta+U(x)\right) \varphi(x)+\int d y V(x-y)|\varphi(y)|^{2} \varphi(x)=\Omega \varphi(x),
$$

где функция $\varphi(x)$ удовлетворяет условию

$$
\int d x|\varphi(x)|^{2}=1
$$

Уравнение Хартри (24) получается из символа, соответствующего обычному вторичному квантованию:

$$
\begin{aligned}
\mathcal{H}_{h}\left[\varphi^{*}(\cdot), \varphi(\cdot)\right]= & \frac{d}{x} \varphi^{*}(x)\left(-\frac{\hbar^{2}}{2 m} \Delta+U(x)\right) \varphi(x)+ \\
& +\frac{1}{2} \iint d x d y V(x-y) \varphi^{*}(x) \varphi^{*}(y) \varphi(y) \varphi(x)
\end{aligned}
$$

Далее рассмотрим случай, когда тор $\mathbf{T}$ моделирует капилляр. Это означает, что мы будем считать, что $L_{1}=L_{2}=l, L_{3}=L, l<L$. Кроме того, рассмотрим сначала случай, когда $U(x)=0$. Уравнение Хартри $(24)$ в таком случае имеет решения вида

$$
\varphi_{k}(x)=\frac{1}{\sqrt{L_{1} L_{2} L_{3}}} e^{i k x}, \quad \Omega_{k}=\frac{\hbar^{2} k^{2}}{2 m}+\frac{\tilde{V}(0)}{L_{1} L_{2} L_{3}}
$$


где

$$
k=\pi\left(\frac{n_{1}}{l}, \frac{n_{2}}{l}, \frac{n_{3}}{L}\right), \quad n_{1}, n_{2}, n_{3} \in \mathbb{Z}, \quad \tilde{V}(k)=\int d x V(x) e^{-i k x} .
$$

Соответствуюшее значение символа (26) равно

$$
\mathcal{H}_{h}\left[\varphi_{k}^{*}(\cdot), \varphi_{k}(\cdot)\right]=\frac{\hbar^{2} k^{2}}{2 m}+\frac{\widetilde{V}(0)}{2 L l^{2}}
$$

Решениям уравнения Хартри вида (27) соответствуют [5], [6] асимптотические серии собственных функций и собственных значений $N$-частичного оператора Гамильтона (6), описывающие текушие состояния системы $N$ частиц. Выражение (29), умноженное на $N$, равно главному члену асимптотики собственных значений $N$-частичного оператора Гамильтона (6), принадлежаших этой серии. Если величина $\hbar|k| / m$ меньше критической скорости Ландау, то такая серия является метастабильной и описывает сверхтекучее состояние. Из эксперимента известно, что на практике сверхтекучесть жидкого гелия исчезает при скоростях, сушественно меньших критической скорости Ландау, за исключением течения через тонкие капилляры. При протекании через капилляры скорость, при которой исчезает сверхтекучесть, зависит от диаметра капилляра и для достаточно узких капилляров совпадает с критической скоростью Ландау. В физике исчезновение сверхтекучести при скоростях, меньших скорости Ландау, объясняется возникновением вихрей. Ниже мы предъявим и исследуем такие решения уравнений (23), которые можно интерпретировать как вихревые решения, а кроме того, дадим объяснение тому факту, что максимальная скорость сверхтекучей жидкости зависит от диаметра капилляра и при некотором его значении становится равна критической скорости Ландау.

В случае, когда $U(x)=0$, уравнения $(23)$ принимают вид

$$
\begin{aligned}
& \Omega \phi(x, y)=-\frac{\hbar^{2}}{2 m}\left(\Delta_{x}+\Delta_{y}\right) \phi(x, y)+ \\
& +\iint d z d w(V(x-y)+V(z-w)) \phi^{+}(z, w)(\phi(x, z) \phi(y, w)+\phi(x, w) \phi(y, z)) \\
& \Omega \phi^{+}(x, y)=-\frac{\hbar^{2}}{2 m}\left(\Delta_{x}+\Delta_{y}\right) \phi^{+}(x, y)+ \\
& +2 \iint d z d w(V(x-z)+V(y-w)) \phi^{+}(x, z) \phi^{+}(y, w) \phi(z, w) .
\end{aligned}
$$

Рассмотрим следуюшие два семейства решений этих уравнений. Первое семейство решений имеет вид

$$
\begin{gathered}
\phi_{1 n}^{+}(x, y)=\frac{1}{l^{2} L} e^{i k_{1 n}(x-y)}, \quad \phi_{1 n}(x, y)=\frac{1}{l^{2} L} \sum_{k} \widetilde{\phi}_{1 n}(k), \\
\Omega_{1 n}=\frac{\hbar^{2} k_{1 n}^{2}}{m}+\frac{2 \widetilde{V}(0)}{l^{2} L}, \\
\widetilde{\phi}_{1 n}(k)=-\frac{1}{4 \widetilde{V}(k)}\left(\frac{\hbar^{2}}{m}\left(k^{2}+k_{1 n}^{2}\right)-\Omega_{1 n}-\sqrt{\left(\frac{\hbar^{2}}{m}\left(k^{2}+k_{1 n}^{2}\right)-\Omega_{1 n}\right)^{2}-4\left(\frac{\widetilde{V}(k)}{l^{2} L}\right)^{2}}\right),
\end{gathered}
$$


где $n$-произвольное целое число, $k_{1 n}$ - вектор вида $2 \pi(0,0, n / L), k$-векторы вида (28), a $\sum_{k}$ обозначает сумму по всем таким векторам. Соответствуюшее (31) значение истинного символа (17) равно

$$
\mathcal{H}\left[\phi_{1 n}^{+}(\cdot), \phi_{1 n}(\cdot)\right]=\frac{\hbar^{2} k_{1 n}^{2}}{2 m}+\frac{\widetilde{V}(0)}{2 l^{2} L} .
$$

Решения (31) уравнений (30) определяют ту же самую асимптотическую серию, что и решения (27) уравнения Хартри (26) при $k=k_{1 n}$. В этом можно убедиться, если построить соответствуюшую асимптотику решения уравнения (21). Кроме того, очевидно, что значения символов (29) и (32) совпадают при $k=k_{1 n}$, т.е. энергии серий, определяемых решениями (27) и (31), в главном порядке по $N$ совпадают. Таким образом, функции (31) соответствуют состоянию, когда система течет вдоль длинной стороны тора $L$ со скоростью $\hbar\left|k_{1 n}\right| / m$.

Второе семейство решений уравнений (30) имеет вид

$$
\begin{gathered}
\phi_{2 n}^{+}(x, y)=\frac{\cos \left(k_{2 n}(x-y)\right)}{l^{2} L}, \quad \phi_{2 n}(x, y)=\frac{1}{l^{2} L} \sum_{k} \widetilde{\phi}_{2 n}(k) e^{i k(x-y)}, \\
\Omega_{2 n}=\frac{\hbar^{2} k_{2 n}^{2}}{m}+\frac{1}{l^{2} L}\left(\widetilde{V}(0)+\widetilde{V}\left(2 k_{2 n}\right)\right), \\
\widetilde{\phi}_{2 n}(k)=-\frac{l^{2} L}{2\left(\widetilde{V}\left(k-k_{2 n}\right)+\widetilde{V}\left(k+k_{2 n}\right)\right)} \times \\
\times\left(\frac{\hbar^{2} k_{2 n}^{2}}{m}-\Omega_{2 n}-\sqrt{\left(\frac{\hbar^{2} k_{2 n}^{2}}{m}-\Omega_{2 n}\right)^{2}-\left(\frac{\widetilde{V}\left(k-k_{2 n}\right)+\widetilde{V}\left(k+k_{2 n}\right)}{l^{2} L}\right)^{2}}\right),
\end{gathered}
$$

где $k_{2 n}$ - вектор вида $2 \pi(n / l, 0,0), n$-произвольное целое неотрицательное число. Соответствуюшее этому решению значение символа (17) равно

$$
\mathcal{H}\left[\phi_{2 n}^{+}(\cdot), \phi_{2 n}(\cdot)\right]=\frac{\hbar^{2} k_{2 n}^{2}}{2 m}+\frac{1}{4 l^{2} L}\left(\widetilde{V}(0)+\widetilde{V}\left(2 k_{2 n}\right)\right)
$$

Решению (33) уравнений (30) отвечает серия, в которой нет течения, среднее значение импульса на состояниях этой серии является величиной порядка $1 / N$. Однако в силу вида функций (33) в состояниях этой серии есть стоячие колебания вдоль одной из коротких сторон тора $l$. Для потенциала $V(x)$ специального вида можно показать, что среди энергий квазичастиц серии, определяемой функциями (33), есть отрицательные, а это означает, что эта серия не является метастабильной и под действием малых возмушений, например трения, состояния этой серии переходят в состояния с меньшей энергией. Поэтому если бы удалось показать, что есть переход из серии, отвечаюшей функциям (31), в серию, отвечаюшую функциям (33), то таким образом можно было бы объяснить исчезновение сверхтекучести при скоростях, меньших критической скорости Ландау. Такой переход возможен, например, если функции (31) и (33) “одинаково 
осциллируют", в квазиклассическом приближении это означает, что пересекаются соответствуюшие этим функциям лагранжевы многообразия. Функции (31) и (33) являются осциллируюшими (квазиклассическими) при $N \rightarrow \infty$, однако из явного вида этих функций следует, что им соответствуют непересекающиеся лагранжевы многообразия. Поэтому далее рассматривается случай, когда есть внешнее поле: $U(x) \neq 0$. Кроме того, рассматривается такой термодинамический передел, при котором в уравнениях (30) автоматически появляется мальй параметр, по которому можно написать квазиклассическую асимптотику решений этих уравнений.

Пусть теперь длины сторон тора Т зависят от числа частиц $N$ следуюшим образом:

$$
l=a N^{1 / 3}, \quad L=b N^{1 / 3},
$$

где $a, b$ - постоянные, не зависяшие от $N$. В этом случае предел при $N \rightarrow \infty$ является термодинамическим пределом, поскольку с увеличением числа частиц тор расширяется так, что плотность частиц остается постоянной. Кроме того, далее мы будем считать, что внешнего поля нет, а потенциал взаимодействия расширяется вместе с тором, т.е. имеет вид

$$
V\left(\frac{x-y}{N^{1 / 3}}\right),
$$

а параметр взаимодействия $\varepsilon$ от $N$ не зависит и равен 1 . Будем считать также, что взаимодействие между бозонами представляет собой отталкивание и $V(x)>0$. Поскольку тор $\mathbf{T}$ становится бесконечно большим при $N \rightarrow \infty$, сделаем в операторе (6) замену переменных $x_{j}=y_{j} N^{1 / 3}, j=1, \ldots, N$, тогда в силу (36) $N$-частичный оператор Гамильтона примет вид

$$
\widehat{H}_{N}=-\frac{\hbar^{2}}{2 m N^{2 / 3}} \sum_{j=1}^{N} \Delta_{j}+\sum_{j=1}^{N} \sum_{l=j+1}^{N} V\left(y_{j}-y_{l}\right),
$$

где переменные $y_{j}, j=1, \ldots, N$, принимают значения на не зависящем от $N$ трехмерном торе $\mathbf{T}$ со сторонами длины $a, a$ и $b$. Оператор $\hat{A}(16)$, отвечающий $(37)$, имеет вид

$$
\begin{aligned}
\widehat{A}= & \iint d x d y \widehat{B}^{+}(x, y)\left(-\frac{\hbar^{2}}{2 m N^{2 / 3}}\left(\Delta_{x}+\Delta_{y}\right)+V(x-y)\right) \widehat{B}^{-}(x, y)+ \\
& +\iiint \int d x d y d z d w V(x-y) \widehat{B}^{+}(x, y) \widehat{B}^{+}(z, w) \times \\
& \times\left(\widehat{B}^{-}(x, z) \widehat{B}^{-}(y, w)+\widehat{B}^{-}(x, w) \widehat{B}^{-}(y, z)\right) .
\end{aligned}
$$

Напишем теперь истинный символ в термодинамическом пределе, для этого рассмотрим символ оператора (38), однако не будем брать предел при $N \rightarrow \infty$ и оставим в символе зависимость от параметра. Получим следуюшее выражение:

$$
\begin{aligned}
\mathcal{H}\left[\phi^{+}(\cdot), \phi(\cdot)\right]= & \iint d x d y \phi^{+}(x, y)\left(-\frac{\hbar^{2}}{2 m N^{2 / 3}}\left(\Delta_{x}+\Delta_{y}\right)+V(x-y)\right) \phi(x, y)+ \\
& +N \iiint \int d x d y d z d w V(x-y) \phi^{+}(x, y) \phi^{+}(z, w) \times \\
& \times(\phi(x, z) \phi(y, w)+\phi(x, w) \phi(y, z))
\end{aligned}
$$


Уравнения (23) для символа (39) записываются следующим образом:

$$
\begin{aligned}
& \Omega \phi(x, y)=\left(-\frac{\hbar^{2}}{2 m N^{2 / 3}}\left(\Delta_{x}+\Delta_{y}\right)+V(x-y)\right) \phi(x, y)+ \\
& \quad+N \iint d z d w(V(x-y)+V(z-w)) \phi^{+}(z, w)(\phi(x, z) \phi(y, w)+\phi(x, w) \phi(y, z)) \\
& \Omega \phi^{+}(x, y)=\left(-\frac{\hbar^{2}}{2 m N^{2 / 3}}\left(\Delta_{x}+\Delta_{y}\right)+V(x-y)\right) \phi^{+}(x, y)+ \\
& \quad+2 N \iint d z d w(V(x-z)+V(y-w)) \phi^{+}(x, z) \phi^{+}(y, w) \phi(z, w) .
\end{aligned}
$$

Кроме того, на решения этой системы уравнений налагается условие (20). Уравнения (40) содержат малый параметр $N^{-1 / 3}$ при первых производных и большой параметр $N$ при интегральном члене. Поэтому будем искать решения этих уравнений в квазиклассическом виде:

$$
\begin{aligned}
\phi(x, y) & =\varphi(x-y) \exp \left(\frac{i}{N^{1 / 3}}(p(x+y)+S(x-y))\right), \\
\phi^{+}(x, y) & =\varphi^{+}(x-y) \exp \left(-\frac{i}{N^{1 / 3}}(p(x+y)+S(x-y))\right),
\end{aligned}
$$

где $\varphi(x), \varphi^{+}(x)$ и $S(x)$ - гладкие функции на торе $\mathbf{T}, p$ - трехмерный вектор, который имеет следующий смысл: $v=p / m$ - скорость течения в рассматриваемой физической системе. Подстановка (41) в уравнения (40) приводит к следуюшей системе уравнений:

$$
\begin{aligned}
\Omega_{0} \varphi(x)= & \frac{\hbar^{2}}{m} \sum_{\alpha=1}^{3}\left(\left(\frac{\partial S}{\partial x^{\alpha}}\right)^{2}(x)-\frac{2 i}{N^{1 / 3}} \frac{\partial S}{\partial x^{\alpha}}(x) \frac{\partial}{\partial x^{\alpha}}-\right. \\
& \left.-\frac{i}{N^{1 / 3}} \frac{\partial^{2} S}{\partial x^{\alpha} \partial x^{\alpha}}(x)-\frac{1}{N^{2 / 3}} \frac{\partial^{2}}{\partial x^{\alpha} \partial x^{\alpha}}\right) \varphi(x)+V(x) \varphi(x)+ \\
& +2 N \iint d \xi d \eta(V(x)+V(x+\xi+\eta)) \varphi^{+}(x+\xi+\eta) \varphi(x+\xi) \varphi(x+\eta) \times \\
& \times \exp \left(i N^{1 / 3}(S(x+\xi)+S(x+\eta)-S(x+\xi+\eta)-S(x))\right) \\
\Omega_{0} \varphi^{+}(x)= & \hbar^{2} \sum_{\alpha=1}^{3}\left(\left(\frac{\partial S}{\partial x^{\alpha}}\right)^{2}(x)+\frac{2 i}{N^{1 / 3}} \frac{\partial S}{\partial x^{\alpha}}(x) \frac{\partial}{\partial x^{\alpha}}+\right. \\
& \left.+\frac{i}{N^{1 / 3}} \frac{\partial^{2} S}{\partial x^{\alpha} \partial x^{\alpha}}(x)-\frac{1}{N^{2 / 3}} \frac{\partial^{2}}{\partial x^{\alpha} \partial x^{\alpha}}\right) \varphi^{+}(x)+V(x) \varphi^{+}(x)+ \\
& +2 N \iint d \xi d \eta(V(x+\xi)+V(x+\eta)) \varphi(x+\xi+\eta) \varphi^{+}(x+\xi) \varphi^{+}(x+\eta) \times \\
& \quad \times \exp \left(-i N^{1 / 3}(S(x+\xi)+S(x+\eta)-S(x+\xi+\eta)-S(x))\right)
\end{aligned}
$$

где

$$
\Omega_{0}=\Omega-\frac{\hbar^{2} p^{2}}{m}
$$


а переменные $\xi$ и $\eta$ лежат на торе $\mathbf{T}$ и интегралы по этим переменным берутся по всему тору. Отметим, что вектор $p$ в уравнениях (42) сократился. Далее будем предполагать, что для функции $S(x)$ выполняется условие

$$
\operatorname{det} D(x) \neq 0 \quad \forall x \in \mathbf{T},
$$

где $D(x)$ - следующая $(3 \times 3)$-матрица:

$$
D_{\alpha \beta}(x)=\frac{\partial^{2} S}{\partial x^{\alpha} \partial x^{\beta}}(x), \quad \alpha, \beta=1,2,3 .
$$

Из условия (44) следует, что лагранжево многообразие в фазовом пространстве $\mathbf{T} \times \mathbb{R}^{3}$

$$
p^{\alpha}=\frac{\partial S}{\partial x^{\alpha}}(x)
$$

взаимно однозначно проецируется на тор Т. Интегралы в уравнениях (42) содержат большой параметр $N^{1 / 3}$ в экспоненте, поэтому их асимптотика при $N \rightarrow \infty$ может быть найдена с помощью метода стационарной фазы. Метод стационарной фазы подробно изложен в работе [7]. Рассмотрим интегралы вида

$$
I(\lambda)=\int d x \chi(x) e^{i \lambda \widetilde{S}(x)}
$$

где $x \in \mathbb{R}^{n}, \widetilde{S}(x)$ - гладкая действительная функция, $\chi(x)$ - гладкая финитная функция, а $\lambda$ - действительный параметр. Согласно методу стационарной фазы, если носитель функции $\chi(x)$ не содержит стационарную точку $x_{0}$ функции $\widetilde{S}(x)$ такую, что

$$
\frac{\partial \widetilde{S}}{\partial x_{0}^{\alpha}}=0 \quad \forall \alpha=1, \ldots, n
$$

то в пределе при $\lambda \rightarrow \infty$ интеграл (47) является величиной, малой по сравнению с любой положительной степенью $1 / \lambda: I(\lambda)=O\left(1 / \lambda^{\infty}\right)$. Если же носитель функции содержит одну стационарную точку $x_{0}$, в которой вьполняется условие

$$
\operatorname{det} \widetilde{D}\left(x_{0}\right) \neq 0
$$

где $\widetilde{D}(x)-(n \times n)$-матрица, соответствуюшая функции $\widetilde{S}(x)$ по формуле $(45)$, то в этом 
случае асимптотика интеграла (47) при $\lambda \rightarrow+\infty$ имеет вид

$$
\begin{aligned}
I(\lambda)= & \left(\frac{2 \pi}{\lambda}\right)^{n / 2} \frac{e^{i \pi M / 4}}{\sqrt{\left|\operatorname{det} \widetilde{D}\left(x_{0}\right)\right|}} e^{i \lambda \widetilde{S}\left(x_{0}\right)} \times \\
& \times\left(\chi\left(x_{0}\right)-\chi\left(x_{0}\right) \frac{i}{8 \lambda} \sum_{\alpha, \beta, \gamma, \delta=1}^{3} \frac{\partial^{4} \widetilde{S}}{\partial x^{\alpha} \partial x^{\beta} \partial x^{\gamma} \partial x^{\delta}}\left(x_{0}\right) \widetilde{D}_{\alpha \beta}^{-1}\left(x_{0}\right) \widetilde{D}_{\gamma \delta}^{-1}\left(x_{0}\right)+\right. \\
& +\chi\left(x_{0}\right) \frac{i}{24 \lambda} \sum_{\alpha, \beta, \gamma=1}^{3} \sum_{\alpha^{\prime}, \beta^{\prime}, \gamma^{\prime}=1}^{3} \frac{\partial^{3} \widetilde{S}}{\partial x^{\alpha} \partial x^{\beta} \partial x^{\gamma}}\left(x_{0}\right) \frac{\partial^{3} \widetilde{S}}{\partial x^{\alpha^{\prime}} \partial x^{\beta^{\prime}} \partial x^{\gamma^{\prime}}}\left(x_{0}\right) \times \\
& \times\left(3 \widetilde{D}_{\alpha \beta}^{-1}\left(x_{0}\right) \widetilde{D}_{\alpha^{\prime} \beta^{\prime}}^{-1}\left(x_{0}\right) \widetilde{D}_{\gamma \gamma^{\prime}}^{-1}\left(x_{0}\right)+2 \widetilde{D}_{\alpha \alpha^{\prime}}^{-1}\left(x_{0}\right) \widetilde{D}_{\beta \beta^{\prime}}^{-1}\left(x_{0}\right) \widetilde{D}_{\gamma \gamma^{\prime}}^{-1}\left(x_{0}\right)\right)- \\
& -\frac{i}{2 \lambda} \sum_{\alpha, \beta, \gamma, \delta=1}^{3} \frac{\partial^{3} \widetilde{S}^{\alpha x^{\alpha} \partial x^{\beta} \partial x^{\gamma}}\left(x_{0}\right) \frac{\partial \chi}{\partial x^{\delta}}\left(x_{0}\right) \widetilde{D}_{\alpha \beta}^{-1}\left(x_{0}\right) \widetilde{D}_{\gamma \delta}^{-1}\left(x_{0}\right)+}{} \\
& \left.+\frac{i}{2 \lambda} \sum_{\alpha, \beta=1}^{3} \frac{\partial^{2} \chi}{\partial x^{\alpha} \partial x^{\beta}} \widetilde{D}_{\alpha \beta}^{-1}\left(x_{0}\right)+O\left(\frac{1}{\lambda^{2}}\right)\right),
\end{aligned}
$$

где $M$ - целое число, равное разности числа положительных и отрицательных собственных значений матрицы $\widetilde{D}\left(x_{0}\right)$, а $\widetilde{D}^{-1}\left(x_{0}\right)$ - матрица, обратная к $\widetilde{D}\left(x_{0}\right)$. Интегралы в формулах (42) имеют вид (47), где $\lambda=N^{1 / 3}$, причем в двух экспонентах с точностью до знака стоит одно и то же действие

$$
\widetilde{S}(\xi, \eta ; x)=S(x+\xi)+S(x+\eta)-S(x+\xi+\eta)-S(x) .
$$

Рассмотрим вопрос о существовании стационарной точки у функции (51). Для каждого заданного $x$ эта точка $\left(\xi_{0}(x), \eta_{0}(x)\right)$ определяется следующей системой уравнений:

$$
\begin{aligned}
\frac{\partial \widetilde{S}}{\partial \xi^{\alpha}}(\xi, \eta ; x) & =\frac{\partial S}{\partial x^{\alpha}}(x+\xi)-\frac{\partial S}{\partial x^{\alpha}}(x+\xi+\eta)=0, \\
\frac{\partial \widetilde{S}}{\partial \eta^{\alpha}}(\xi, \eta ; x) & =\frac{\partial S}{\partial x^{\alpha}}(x+\eta)-\frac{\partial S}{\partial x^{\alpha}}(x+\xi+\eta)=0 \quad \forall \alpha=1,2,3 .
\end{aligned}
$$

Очевидно, что эти уравнения удовлетворяются при $\xi=\eta=(0,0,0)$, а в силу условия (44) другого решения у этих уравнений не может быть. Таким образом, для нахождения асимптотики интегралов в формулах (42) применима формула (50). Подставим (50) в (42) и приравняем члены, не содержашие параметра $N^{-1 / 3}$ в левой и правой частях уравнений. Получим следующее уравнение Гамильтона-Якоби для действия $S(x)$ :

$$
\frac{\hbar^{2}}{m} \sum_{\alpha=1}^{3}\left(\frac{\partial S}{\partial x^{\alpha}}\right)^{2}(x)+V(x)+\frac{4 V(x) \rho(x)}{|\operatorname{det}(D(x) /(2 \pi))|}=\Omega_{0},
$$

где

$$
\rho(x)=\lim _{N \rightarrow \infty} \varphi^{+}(x) \varphi(x) .
$$


Чтобы найти уравнение для величины (54), после подстановки (50) умножим правую часть первого из уравнений $(42)$ на $\varphi^{+}(x)$, а левую часть второго - на $\varphi(x)$, после чего вычтем эти выражения одно из другого и в ответе приравняем нулю коэффициент при $N^{-1 / 3}$. В результате получим уравнение вида

$$
\sum_{\alpha=1}^{3} \frac{\partial}{\partial x^{\alpha}}\left(\frac{\hbar^{2}}{m} \frac{\partial S}{\partial x^{\alpha}}(x) \rho(x)+\sum_{\beta=1}^{3} \frac{\partial}{\partial x^{\beta}}\left(\frac{V(x)(\rho(x))^{2} D_{\alpha \beta}^{-1}(x)}{|\operatorname{det}(D(x) /(2 \pi))|}\right)\right)=0 .
$$

Заметим, что из системы уравнений $(53),(55)$ можно исключить функцию $\rho(x)$, выразив ее через $\partial S / \partial x$ согласно уравнению (53) и подставив результат в (55). Заметим также, что из $(20)$ и $(41)$ следует условие для $\rho(x)$ :

$$
\int d x \rho(x)=\frac{1}{2}
$$

Уравнения (53), (55) сложны для исследования, поэтому рассмотрим более простой одномерный случай, когда частицы находятся на окружности. В одномерном случае в уравнениях (42) малый параметр при производных будет $1 / N$, и соответственно этот же параметр надо поставить в $(41)$ вместо $N^{-1 / 3}$. Применяя метод стационарной фазы описанным выше способом в одномерном случае получим уравнения

$$
\begin{aligned}
\frac{\hbar^{2}}{m}\left(S^{\prime}(x)\right)^{2}+V(x)+\frac{8 \pi V(x) \rho(x)}{\left|S^{\prime \prime}(x)\right|} & =\Omega_{0}, \\
\frac{d}{d x}\left(\frac{\hbar^{2}}{m} S^{\prime}(x) \rho(x)+2 \pi \frac{d}{d x}\left(\frac{V(x)(\rho(x))^{2}}{S^{\prime \prime}(x)\left|S^{\prime \prime}(x)\right|}\right)\right) & =0 .
\end{aligned}
$$

Из (57) получим

$$
\rho(x)=\frac{\Omega_{0}-\hbar^{2}\left(S^{\prime}(x)\right)^{2} / m-V(x)}{8 \pi V(x)}\left|S^{\prime \prime}(x)\right| .
$$

Подстановка $(59)$ в (58) окончательно дает следующее уравнение для $S(x)$ :

$$
\frac{d}{d x}\left(\frac{V^{\prime}(x)}{(V(x))^{2}}\left(\left(\Omega_{0}-\frac{\hbar^{2}}{m}\left(S^{\prime}(x)\right)^{2}\right)^{2}-(V(x))^{2}\right)\right)=0 .
$$

Это уравнение решается относительно $S^{\prime}(x)$ :

$$
S^{\prime}(x)= \pm \sqrt{\frac{m}{\hbar^{2}}} \sqrt{\Omega_{0}-V(x) \sqrt{\frac{C}{V^{\prime}(x)}+1}}
$$

где $C$ - число, которое определяется из условия (56). Так как в случае отталкивания $V(x)>0$, то решение (61) не может сушествовать на всей окружности, если $V^{\prime}(x)$ обращается в нуль в какой-то точке на окружности. Однако для гладкого потенциала $V(x)$ на окружности сушествуют по крайней мере две точки, где его производная равна нулю. 
Таким образом, получается, что в одномерном случае для системы бозонов с отталкиванием не могут существовать квазиклассические решения системы уравнений (42), соответствующие лагранжеву многообразию, взаимно однозначно проецирующемуся на координатную окружность фазового пространства. Поэтому в одномерном случае квазиклассические решения уравнений (42) обязательно содержат фокальную точку, причем в этой точке в силу (57) $S^{\prime}(x)=0$. Это означает, что соответствующая функция $\phi(x, y)$ такова, что ее интеграл и матричные элементы с единичной функцией не являются экспоненциально малыми при $N \rightarrow \infty$, а это, в свою очередь, означает возможность перехода из боголюбовского состояния [6] в рассматриваемое здесь парное несверхтекучее состояние. Такой переход приводит к исчезновению сверхтекучести.

В двумерном и трехмерном случаях получить точное решение, аналогичное решению в одномерном случае, непосредственно не удается. Может быть, соответствуюшие уравнения в этих случаях могут быть решены численно или аналитически с помощью компьютера.

Рассмотрим случай, когда в ультравторичном квантовании кроме пар участвуют также одиночные частицы. В этом случае также имеет место тождество (15), где оператор $\widehat{A}$ имеет вид

$$
\begin{aligned}
\widehat{A}= & \int d x \hat{b}^{+}(x)\left(-\frac{\hbar^{2}}{2 m} \Delta_{x}\right) \hat{b}^{-}(x)+\frac{\varepsilon}{2} \iint d x d y V(x-y) \hat{b}^{+}(x) \hat{b}^{+}(y) \hat{b}^{-}(y) \hat{b}^{-}(x)+ \\
& +\iint d x d y \widehat{B}^{+}(x, y)\left(-\frac{\hbar^{2}}{2 m}\left(\Delta_{x}+\Delta_{y}\right)+U(x)+U(y)+\varepsilon V(x-y)\right) \widehat{B}^{-}(x, y)+ \\
& +\varepsilon \iiint \int d x d y d z d w V(x-y) \widehat{B}^{+}(x, y) \widehat{B}^{+}(z, w) \times \\
& \times\left(\widehat{B}^{-}(x, z) \widehat{B}^{-}(y, w)+\widehat{B}^{-}(x, w) \widehat{B}^{-}(y, z)\right)+ \\
& +\varepsilon \iiint d x d y d z V(x-y)\left(a \hat{b}^{+}(x) \widehat{B}^{+}(y, z)+b \hat{b}^{+}(z) \widehat{B}^{+}(x, y)\right) \times \\
& \times\left(\widehat{B}^{-}(x, z) \hat{b}^{-}(y)+\widehat{B}^{-}(y, z) \hat{b}^{-}(x)\right)
\end{aligned}
$$

где $\hat{b}^{ \pm}(x)$ - операторы рождения и уничтожения одиночных частиц, а $a, b$ - произвольные числа такие, что $a+b=1$, т.е. сушествует произвол в выборе истинного символа в этом случае. Оператор числа частиц (19) с учетом одиночных частиц примет вид

$$
\widehat{N}=\int d x \hat{b}^{+}(x) \hat{b}^{-}(x)+2 \iint d x d y \widehat{B}^{+}(x, y) \widehat{B}^{-}(x, y) .
$$

Для оператора (62) можно проделать все то же, что и для оператора (16). В частности, если перейти в $(62)$ от операторов $\hat{b}^{ \pm}(x)$ и $\widehat{B}^{ \pm}(x, y)$ к символам (символы одиночных частиц будем обозначать $f^{+}(x)$ и $\left.f(x)\right)$, то можно написать уравнения, аналогичные уравнениям (40). Пусть параметр взаимодействия $\varepsilon$ равен 1 , а потенциал взаимодействия не расширяется вместе с тором. Тогда уравнения (40) с учетом одиночных частиц 
примут вид

$$
\begin{aligned}
& \Omega f(x)=-\frac{\hbar^{2}}{2 m N^{2 / 3}} \Delta f(x)+N \int d y V\left(N^{1 / 3}(x-y)\right)|f(y)|^{2} f(x)+ \\
& +N a \iint d y d z V\left(N^{1 / 3}(x-y)\right) \phi^{+}(y, z)(\phi(x, z) f(y)+\phi(y, z) f(x))+ \\
& +N b \iint d y d z V\left(N^{1 / 3}(z-y)\right) \phi^{+}(y, z)(\phi(x, z) f(y)+\phi(x, y) f(z)), \\
& \Omega f^{+}(x)=-\frac{\hbar^{2}}{2 m N^{2 / 3}} \Delta f^{+}(x)+N \int d y V\left(N^{1 / 3}(x-y)\right)|f(y)|^{2} f^{+}(x)+ \\
& +N \iint d y d z V\left(N^{1 / 2}(x-y)\right) \phi(y, z)\left(a \phi^{+}(y, z) f^{+}(x)+b \phi^{+}(x, y) f^{+}(z)\right)+ \\
& +N \iint d y d z V\left(N^{1 / 3}(x-y)\right) \phi(y, z)\left(a \phi^{+}(x, z) f^{+}(y)+b \phi^{+}(x, y) f^{+}(z)\right)
\end{aligned}
$$

и

$$
\begin{aligned}
2 \Omega \phi(x, & y)=\left(-\frac{\hbar^{2}}{2 m N^{2 / 3}}\left(\Delta_{x}+\Delta_{y}\right)+V\left(N^{1 / 3}(x-y)\right)\right) \phi(x, y)+ \\
& +2 N \iint d z d w\left(V\left(N^{1 / 3}(x-y)\right)+V\left(N^{1 / 3}(z-w)\right)\right) \phi^{+}(z, w) \phi(x, z) \phi(y, w)+ \\
& +\frac{N a}{2} \int d z V\left(N^{1 / 3}(y-z)\right) f^{+}(z)(\phi(x, z) f(y)+\phi(x, y) f(z))+ \\
& +\frac{N a}{2} \int d z V\left(N^{1 / 3}(x-z)\right) f^{+}(z)(\phi(y, z) f(x)+\phi(x, y) f(z))+ \\
& +N b \int d z V\left(N^{1 / 3}(x-y)\right) f^{+}(z)(\phi(x, z) f(y)+\phi(y, z) f(x)), \\
2 \Omega \phi^{+} & \left.x, y)=\int-\frac{\hbar^{2}}{2 m N^{2 / 3}}\left(\Delta_{x}+\Delta_{y}\right)+V\left(N^{1 / 3}(x-y)\right)\right) \phi^{+}(x, y)+ \\
& +2 N \iint d z d w\left(V\left(N^{1 / 3}(x-z)\right)+V\left(N^{1 / 3}(y-w)\right)\right) \phi(z, w) \phi^{+}(x, z) \phi^{+}(y, w)+ \\
& +\frac{N}{2} \int d z V\left(N^{1 / 3}(y-z)\right) f(z) \times \\
& \times\left(a \phi^{+}(x, z) f^{+}(y)+a \phi^{+}(x, y) f^{+}(z)+2 b \phi^{+}(y, z) f^{+}(x)\right)+ \\
& +\frac{N}{2} \int d z V\left(N^{1 / 3}(x-z)\right) f(z) \times \\
& \times\left(a \phi^{+}(y, z) f^{+}(x)+a \phi^{+}(x, y) f^{+}(z)+2 b \phi^{+}(x, z) f^{+}(y)\right) .
\end{aligned}
$$

Система уравнений (64), (65) допускает подстановку

$$
\begin{aligned}
f(x) & =\sqrt{n_{0}} e^{i N^{1 / 3} p x}, & f^{+}(x) & =\sqrt{n_{0}} e^{-i N^{1 / 3} p x}, \\
\phi(x, y) & =e^{i N^{1 / 3} p(x+y)} \varphi(x-y), & \phi^{+}(x, y) & =e^{-i N^{1 / 3} p(x+y)} \varphi^{+}(x-y),
\end{aligned}
$$


которая приводит к следующей системе уравнений:

$$
\begin{aligned}
& \Omega_{0}=N n_{0} \int d x V\left(N^{1 / 3} x\right)+2 N b \iint d y d z V\left(N^{1 / 3} y\right) \varphi^{+}(y) \varphi(z)+ \\
& +N a \iint d y d z V\left(N^{1 / 3} y\right)\left(\varphi^{+}(z) \varphi(z)+\varphi^{+}(z) \varphi(z-y)\right), \\
& 2 \Omega_{0} \varphi(x)=\left(-\frac{\hbar^{2}}{m N^{2 / 3}} \Delta+V\left(N^{1 / 3} x\right)\right) \varphi(x)+ \\
& +2 N \iint d z d w\left(V\left(N^{1 / 3} x\right)+V\left(N^{1 / 3}(x+z-w)\right)\right) \times \\
& \times \varphi^{+}(x+z-w) \varphi(z) \varphi(w)+2 N b n_{0} \int d z V\left(N^{1 / 3} x\right) \varphi(z)+ \\
& +\operatorname{Nan}_{0} \int d z\left(V\left(N^{1 / 3}(x+z)\right) \varphi(z)+V\left(N^{1 / 3} z\right) \varphi(x)\right), \\
& 2 \Omega_{0} \varphi^{+}(x)=\left(-\frac{\hbar^{2}}{m N^{2 / 3}} \Delta+V\left(N^{1 / 3} x\right)\right) \varphi^{+}(x)+ \\
& +2 N \iint d z d w\left(V\left(N^{1 / 3} z\right)+V\left(N^{1 / 3} w\right)\right) \varphi(x+z-w) \varphi^{+}(z) \varphi^{+}(w)+ \\
& +2 N b n_{0} \int d z V\left(N^{1 / 3} z\right) \varphi^{+}(z)+ \\
& +\operatorname{Nan}_{0} \int d z\left(V\left(N^{1 / 3}(x+z)\right) \varphi^{+}(z)+V\left(N^{1 / 3} z\right) \varphi^{+}(x)\right),
\end{aligned}
$$

где $n_{0}$ - доля одиночных среди всех частиц, а $\Omega_{0}=\Omega-\hbar^{2} p^{2} /(2 m)$. К системе уравнений (67), так же как к (40), применимы квазиклассические методы, однако в этом случае для потенциала взаимодействия может быть использован метод стационарной фазы, поскольку он является быстро убываюшим. Квазиклассическую асимптотику решений системы (67) можно написать, например, в случае, когда потенциал взаимодействия имеет вид $V(x)=U e^{-c|x|^{d}}$, где $c>0$ и $0<d<1$. Заметим, наконец, что уравнения (67) отличаются от уравнений (40) наличием двух параметров - $n_{0}$ и $a, b=1-a$, что дает больше возможностей для нахождения толшины капилляра, при которой исчезает сверхтекучесть.

Рассмотрим систему $N$ тождественных бозонов массы $m$, которые находятся на трехмерном торе $\mathbf{T}$, длины сторон которого равны $L_{1}, L_{2}$ и $L_{2}$. Будем считать, что бозоны взаимодействуют между собой и потенциал взаимодействия имеет вид

$$
V\left(N^{1 / 3}(x-y)\right)
$$

где $V(\xi)$ - гладкая четная функция, быстро убывающая на бесконечности, $x, y$ - координаты бозонов на торе Т. Потенциал взаимодействия (68) зависит от $N$ таким образом, что его радиус уменьшается с увеличением числа частиц $N$, при этом в среднем число частиц, с которыми взаимодействует одна частица, остается постоянным.

При ультравторичном квантовании по парам рассматриваемой бозонной системе соответствует ультравторично-квантованный оператор $\widehat{\widehat{H}}$, явный вид которого приведен 
ранее. Как обсуждалось выше, для этого ультравторично-квантованного оператора имеет место тождество (15), где $\widehat{\widehat{E}}$ - ультравторично-квантованный единичный оператор, а $\widehat{A}$ - оператор в пространстве ультравторичного квантования, выбор которого неоднозначен. Далее мы считаем, что выбран оператор $\widehat{A}$ вида

$$
\begin{aligned}
\widehat{A}= & \iint d x d y \widehat{B}^{+}(x, y)\left(-\frac{\hbar^{2}}{2 m}\left(\Delta_{x}+\Delta_{y}\right)+V\left(N^{1 / 3}(x-y)\right)\right) \widehat{B}^{-}(x, y)+ \\
& +2 \int d x d y d x^{\prime} d y^{\prime} V\left(N^{1 / 3}(x-y)\right) \widehat{B}^{+}(x, y) \widehat{B}^{+}\left(x^{\prime}, y^{\prime}\right) \widehat{B}^{-}\left(x, x^{\prime}\right) \widehat{B}^{-}\left(y, y^{\prime}\right),
\end{aligned}
$$

где $\widehat{B}^{+}(x, y)$ и $\widehat{B}^{-}(x, y)$ - соответственно бозонные операторы рождения и уничтожения пары частиц в фоковском пространстве ультравторичного квантования. В силу тождества (15) для нахождения асимптотики спектра рассматриваемой бозонной системы в пределе при $N \rightarrow \infty$ можно найти соответствуюшую асимптотику для оператора (69).

Так как функция (68), умноженная на $N$, в пределе при $N \rightarrow \infty$ в некотором смысле сходится к дельта-функции Дирака, то в операторе (69) при втором слагаемом в этом предельном случае стоит малый параметр $1 / N$. Это означает, что для нахождения асимптотики собственных значений и собственных функций оператора $\widehat{A}$ можно применить квазиклассические методы, развитые в [8]. Асимптотика собственных значений и собственных функций определяется символом оператора (69), этот символ называется истинным символом ультравторично-квантованной задачи. Истинным символом, соответствующим оператору (69), является следующий функционал, определенный для пары функций $\Phi^{+}(x, y)$ и $\Phi(x, y)$ :

$$
\begin{aligned}
& \mathcal{H}\left[\Phi^{+}(\cdot), \Phi(\cdot)\right]=\iint d x d y \Phi^{+}(x, y)\left(-\frac{\hbar^{2}}{2 m}\left(\Delta_{x}+\Delta_{y}\right)\right) \Phi(x, y)+ \\
& \quad+2 \iiint \int d x d y d x^{\prime} d y^{\prime} N V\left(N^{1 / 3}(x-y)\right) \Phi^{+}(x, y) \Phi^{+}\left(x^{\prime}, y^{\prime}\right) \Phi\left(x, x^{\prime}\right) \Phi\left(y, y^{\prime}\right) .
\end{aligned}
$$

Из сохранения числа частиц в системе для функций $\Phi^{+}(x, y)$ и $\Phi(x, y)$ следует условие

$$
\iint d x d y \Phi^{+}(x, y) \Phi(x, y)=\frac{1}{2}
$$

Согласно асимптотическим методам [8] каждому решению системы уравнений

$$
\Omega \Phi(x, y)=\frac{\delta \mathcal{H}}{\delta \Phi^{+}(x, y)}, \quad \Omega \Phi^{+}(x, y)=\frac{\delta \mathcal{H}}{\delta \Phi(x, y)}
$$

удовлетворяющему также условию (71), в пределе при $N \rightarrow \infty$ соответствует асимптотическая серия собственных функций и собственных значений оператора (69). Из явного 
вида истинного символа (70) следует, что система уравнений (72) записывается в виде

$$
\begin{aligned}
\Omega \Phi(x, y)= & -\frac{\hbar^{2}}{2 m}\left(\Delta_{x}+\Delta_{y}\right) \Phi(x, y)+ \\
& +\iint d x^{\prime} d y^{\prime}\left(N V\left(N^{1 / 3}(x-y)\right)+N V\left(N^{1 / 3}\left(x^{\prime}-y^{\prime}\right)\right)\right) \times \\
& \times \Phi^{+}\left(x^{\prime}, y^{\prime}\right) \Phi\left(x, x^{\prime}\right) \Phi\left(y, y^{\prime}\right), \\
\Omega \Phi^{+}(x, y)=- & \frac{\hbar^{2}}{2 m}\left(\Delta_{x}+\Delta_{y}\right) \Phi^{+}(x, y)+ \\
& +\iint d x^{\prime} d y^{\prime}\left(N V\left(N^{1 / 3}\left(x-x^{\prime}\right)\right)+N V\left(N^{1 / 3}\left(y-y^{\prime}\right)\right)\right) \times \\
& \times \Phi\left(x^{\prime}, y^{\prime}\right) \Phi^{+}\left(x, x^{\prime}\right) \Phi^{+}\left(y, y^{\prime}\right) .
\end{aligned}
$$

В пределе при $N \rightarrow \infty$ эта система уравнений, дополненная условием $(71)$, имеет следующее семейство решений:

$$
\begin{aligned}
& \Phi_{k_{1}, k_{2}}^{+}(x, y)=\frac{1}{L_{1} L_{2}^{2}} e^{i k_{1}(x+y)} \cos \left(k_{2}(x-y)\right), \\
& \Phi_{k_{1}, k_{2}}(x, y)=\frac{1}{L_{1} L_{2}^{2}} \sum_{l} \varphi_{k_{2}, l} e^{i\left(l+k_{1}\right)(x-y)},
\end{aligned}
$$

где $k_{1}, k_{2}$ и $l$ - трехмерные векторы вида

$$
2 \pi\left(\frac{n_{1}}{L_{1}}, \frac{n_{2}}{L_{2}}, \frac{n_{3}}{L_{2}}\right)
$$

$n_{1}, n_{2}, n_{3}$ - целые числа, $\varphi_{k_{2}, l}$ имеют вид

$$
\varphi_{k_{2}, l}=\frac{1}{2 V_{0}}\left(\frac{\hbar^{2}}{2 m}\left(k_{2}^{2}-l^{2}\right)+V_{0} \pm \sqrt{\left(\frac{\hbar^{2}}{2 m}\left(k_{2}^{2}-l^{2}\right)+V_{0}\right)^{2}-V_{0}^{2}}\right)
$$

где выбирается знак плюс при $l^{2}>k_{2}^{2}$ и знак минус при $l^{2}<k_{2}^{2}$, а $V_{0}$ здесь и далее обозначает величину

$$
V_{0}=\frac{1}{L_{1} L_{2}^{2}} \int V(x) d x
$$

где интеграл берется по пространству $\mathbb{R}^{3}$. Пара векторов $k_{1}, k_{2}$ в формулах $(74)$ играет роль параметров, нумеруюших различные решения системы уравнений (73), (71). Причем вектор $k_{1}$ имеет ясный физический смысл: величина $\hbar k_{1} / m$ равна скорости течения бозонной системы.

Главный член асимптотики собственных значений серии, соответствуюшей решению (74), равен значению символа (70) на функциях (74), умноженному на $N$ :

$$
E_{k_{1}, k_{2}}=N\left(\frac{\hbar^{2}\left(k_{1}^{2}+k_{2}^{2}\right)}{2 m}+\frac{V_{0}}{2}\right) .
$$


Асимптотика собственных значений и собственных функций, в частности следуюшие после (78) члены, помимо решений системы уравнений (73), определяется также решениями системы уравнений в вариациях для системы уравнений (73). Система уравнений в вариациях для (73) имеет вид

$$
\begin{aligned}
(\Omega-\lambda) F(x, y)= & -\frac{\hbar^{2}}{2 m}\left(\Delta_{x}+\Delta_{y}\right) F(x, y)+ \\
& +\iint d x^{\prime} d y^{\prime}\left(N V\left(N^{1 / 3}(x-y)\right)+N V\left(N^{1 / 3}\left(x^{\prime}-y^{\prime}\right)\right)\right) \times \\
& \times\left(G\left(x^{\prime}, y^{\prime}\right) \Phi\left(x, x^{\prime}\right) \Phi\left(y, y^{\prime}\right)+\right. \\
& \left.+\Phi^{+}\left(x^{\prime}, y^{\prime}\right) F\left(x, x^{\prime}\right) \Phi\left(y, y^{\prime}\right)+\Phi^{+}\left(x^{\prime}, y^{\prime}\right) \Phi\left(x, x^{\prime}\right) F\left(y, y^{\prime}\right)\right), \\
(\Omega+\lambda) G(x, y)= & -\frac{\hbar^{2}}{2 m}\left(\Delta_{x}+\Delta_{y}\right) G(x, y)+ \\
& +\iint d x^{\prime} d y^{\prime}\left(N V\left(N^{1 / 3}\left(x-x^{\prime}\right)\right)+N V\left(N^{1 / 3}\left(y-y^{\prime}\right)\right)\right) \times \\
& \times\left(F\left(x^{\prime}, y^{\prime}\right) \Phi^{+}\left(x, x^{\prime}\right) \Phi^{+}\left(y, y^{\prime}\right)+\right. \\
& \left.+\Phi\left(x^{\prime}, y^{\prime}\right) G\left(x, x^{\prime}\right) \Phi^{+}\left(y, y^{\prime}\right)+\Phi\left(x^{\prime}, y^{\prime}\right) \Phi^{+}\left(x, x^{\prime}\right) G\left(y, y^{\prime}\right)\right) .
\end{aligned}
$$

Для нахождения спектра квазичастиц из всех решений системы уравнений в вариациях (79) надо выделить те, которые удовлетворяют правилу отбора:

$$
\iint d x d y\left(G^{*}(x, y) G(x, y)-F^{*}(x, y) F(x, y)\right)>0 .
$$

Кроме того, надо учесть, что в формуле (15) стоит оператор проектирования на симметричное подпространство $\widehat{\widehat{E}}$, это приводит, в частности, к тому, что надо рассматривать симметричные решения системы уравнений в вариациях:

$$
F(x, y)=F(y, x), \quad G(x, y)=G(y, x)
$$

Если $k_{2}=0$, то соответствуюшая этому решению (74) системы уравнений (73), (71) асимптотическая серия является боголюбовской серией, отвечаюшей скорости течения $\hbar k_{1} / m$. Спектр квазичастиц этой серии выражается хорошо известной формулой

$$
\lambda_{l}=\sqrt{\left(\frac{\hbar^{2} l^{2}}{2 m}+V_{0}\right)^{2}-V_{0}^{2}}-\frac{\hbar^{2} l k_{1}}{m} .
$$

Рассмотрим теперь случай, когда $k_{2} \neq 0$. Подставим $(74)$ в $(79)$ и учтем симметрию и то, что потенциал взаимодействия зависит от разности координат. Получим, что ре- 
шения системы уравнений в вариациях (79) в этом случае имеют вид

$$
\begin{aligned}
F_{l}(x, y)= & \frac{u_{1, l}}{2}\left(\exp \left(i\left(k_{1}+k_{2}\right) x+i\left(k_{1}+l\right) y\right)+\exp \left(i\left(k_{1}+k_{2}\right) y+i\left(k_{1}+l\right) x\right)\right)+ \\
& +\frac{u_{2, l}}{2}\left(\exp \left(i\left(k_{1}-k_{2}\right) x+i\left(k_{1}+2 k_{2}+l\right) y\right)+\right. \\
& \left.+\exp \left(i\left(k_{1}-k_{2}\right) y+i\left(k_{1}+2 k_{2}+l\right) x\right)\right) \\
G_{l}(x, y)= & \frac{v_{1, l}}{2}\left(\exp \left(i\left(k_{1}+k_{2}\right) x+i\left(k_{1}+l\right) y\right)+\exp \left(i\left(k_{1}+k_{2}\right) y+i\left(k_{1}+l\right) x\right)\right)+ \\
& +\frac{v_{2, l}}{2}\left(\exp \left(i\left(k_{1}-k_{2}\right) x+i\left(k_{1}+2 k_{2}+l\right) y\right)+\right. \\
& \left.+\exp \left(i\left(k_{1}-k_{2}\right) y+i\left(k_{1}+2 k_{2}+l\right) x\right)\right)+ \\
& +\sum_{l^{\prime} \neq l, l+2 k_{2}} \frac{w_{l, l^{\prime}}}{2}\left(\exp \left(i\left(k_{1}+k_{2}+l-l^{\prime}\right) x+i\left(k_{1}+l^{\prime}\right) y\right)+\right. \\
& \left.+\exp \left(i\left(k_{1}+k_{2}+l-l^{\prime}\right) y+i\left(k_{1}+l^{\prime}\right) x\right)\right),
\end{aligned}
$$

где $l \neq-k_{2}$ - трехмерный вектор вида (75), а числовые коэффициенты $u_{1, l}, u_{2, l}, v_{1, l}$, $v_{2, l}$ и $w_{l, l^{\prime}}, l^{\prime} \neq l$, определяются из бесконечной, вообше говоря, системы уравнений. Отметим, однако, что бесконечная система уравнений содержит замкнутую подсистему из четырех уравнений для коэффициентов $u_{1, l}, u_{2, l}, v_{1, l}$ и $v_{2, l}$, эта система может быть записана в следуюшем, стандартном для линейной алгебры виде:

$$
\widetilde{\lambda} X=M X
$$

где

$$
\widetilde{\lambda}=\lambda-\frac{\hbar^{2}}{m} k_{1}\left(k_{2}+l\right),
$$

$X-$ вектор-столбец вида

$$
X=\left(\begin{array}{l}
u_{1, l} \\
u_{2, l} \\
v_{1, l} \\
v_{2, l}
\end{array}\right)
$$

а $M-(4 \times 4)$-матрица:

$$
M=\left(\begin{array}{cccc}
B & V_{0} & -V_{0} & 0 \\
V_{0} & B_{1} & 0 & -V_{0} \\
2 V_{0} \varphi_{k_{2}, l} & V_{0}\left(\varphi_{k_{2}, l}+\varphi_{k_{2}, l+2 k_{2}}\right) & -B & -V_{0} \\
V_{0}\left(\varphi_{k_{2}, l}+\varphi_{k_{2}, l+2 k_{2}}\right) & 2 V_{0} \varphi_{k_{2}, l+2 k_{2}} & -V_{0} & -B_{1}
\end{array}\right)
$$

$B$ и $B_{1}$ имеют вид

$$
B=\frac{\hbar^{2}}{2 m}\left(l^{2}-k_{2}^{2}\right)+V_{0} \varphi_{k_{2}, l}, \quad B_{1}=\frac{\hbar^{2}}{2 m}\left(\left(l+2 k_{2}\right)^{2}-k_{2}^{2}\right)+V_{0} \varphi_{k_{2}, l+2 k_{2}},
$$

a $V_{0}$ и $\varphi_{k_{2}, l}$ выражаются формулами $(77)$ и (76), соответственно. Из (80) и (83) следует правило отбора для решений системы уравнений (84):

$$
u_{1, l}^{*} u_{1, l}+u_{2, l}^{*} u_{2, l}-v_{1, l}^{*} v_{1, l}-v_{2, l}^{*} v_{2, l}>0 .
$$


Система линейных уравнений (84) была решена на компьютере с помошью программы Maple, правила отбора (85) выделяют следующие собственные значения:

$$
\begin{aligned}
& \lambda_{1, k_{1}, k_{2}, l}=-\frac{\hbar^{2}}{m} k_{1}\left(k_{2}+l\right) \pm \\
& \pm \frac{\hbar^{2}}{2 m} \sqrt{k_{2}^{4}+\frac{l^{2}}{2}+\frac{l_{1}^{2}}{2}-k_{2}^{2} l^{2}-k_{2}^{2} l_{1}^{2}+\frac{1}{2}\left(l^{2}+l_{1}^{2}-2 k_{2}^{2}\right) \sqrt{\left(l^{2}-l_{1}^{2}\right)^{2}+\left(\frac{4 m V_{0}}{\hbar^{2}}\right)^{2}}} \\
& \lambda_{2, k_{1}, k_{2}, l}=-\frac{\hbar^{2}}{m} k_{1}\left(k_{2}+l\right) \pm \\
& \pm \frac{\hbar^{2}}{2 m} \sqrt{k_{2}^{4}+\frac{l^{2}}{2}+\frac{l_{1}^{2}}{2}-k_{2}^{2} l^{2}-k_{2}^{2} l_{1}^{2}-\frac{1}{2}\left(l^{2}+l_{1}^{2}-2 k_{2}^{2}\right) \sqrt{\left(l^{2}-l_{1}^{2}\right)^{2}+\left(\frac{4 m V_{0}}{\hbar^{2}}\right)^{2}}}
\end{aligned}
$$

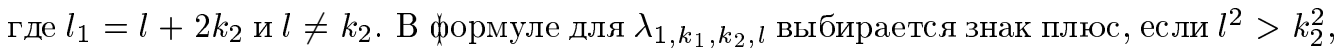
и знак минус, если $l^{2}<k_{2}^{2}$, а в формуле для $\lambda_{2, k_{1}, k_{2}, l}-$ знак плюс, если $l_{1}^{2}>k_{2}^{2}$, и знак минус, если $l_{1}^{2}<k_{2}^{2}$.

Формулы (86) при $l \neq-k_{2}$ задают спектр квазичастиц серии, отвечающей решению (74). Из явного вида (86) следует, что в этом спектре квазичастиц есть отрицательные значения, а значит, серия, соответствуюшая решению (74) при $k \neq 0$, не является метастабильной. В связи с этим предлагается следуюшее объяснение зависимости критической скорости от ширины капилляра. Будем считать далее, что $L_{1} \gg L_{2}$. Рассмотрим боголюбовскую серию, соответствующую течению вдоль капилляра со скоростью $\hbar k_{0} / m$, где $k_{0}=2 \pi\left(n_{1} / L_{1}, 0,0\right)$. Для рассматриваемой здесь системы бозонов главный член асимптотики собственных значений этой серии равен

$$
N\left(\frac{\hbar^{2} k_{0}^{2}}{2 m}+\frac{V_{0}}{2}\right) .
$$

Допустим теперь, что соотношение между $L_{1}$ и $L_{2}$ такое, что сушествует пара векторов $k_{1}$ и $k_{2}=2 \pi\left(0, n_{2} / L_{2}, n_{3} / L_{2}\right)$ такая, что соответствующее ей значение члена (78) в точности равно (87). Это означает, что между текучими состояниями боголюбовской серии и состояниями неметастабильной серии, соответствуюшей решению (74), возможен резонанс. Если значение $L_{1}$ очень велико, резонанс также возможен в случае, когда значение члена (78) близко к (25) и необязательно точно совпадает с ним. Сушествование такого резонанса означает возможность перехода из текучего состояния в неметастабильное, из которого система упадет на нижний энергетический уровень, что означает потерю сверхтекучести. Минимальная энергия, отвечающая неметастабильной серии, соответствует случаю $k_{2}=2 \pi\left(0,1 / L_{2}, 0\right)$ и согласно формуле $(78)$ равна

$$
E_{\min }=N\left(\frac{\hbar^{2}(2 \pi)^{2}}{2 m L_{2}^{2}}+\frac{V_{0}}{2}\right)
$$


Сравнивая (88) с (87), получаем, что резонанс невозможен, если скорость течения $v$ по модулю меньше некоторой величины:

$$
|v|<v_{\mathrm{c}}\left(L_{2}\right) \equiv \frac{2 \pi \hbar}{m L_{2}} .
$$

Величина $v_{\mathrm{c}}\left(L_{2}\right)$ в правой части неравенства (89) увеличивается с уменьшением толшины капилляра $L_{2}$. Если $L_{2}$ меньше величины $2 \pi \hbar /\left(m v_{\mathrm{cL}}\right)$, где $v_{\mathrm{cL}}-$ критическая скорость Ландау, определяемая с помошью $(82)$, то $v_{\mathrm{c}}\left(L_{2}\right)$ больше, чем критическая скорость Ландау, и соответственно в этом случае сверх текучесть исчезает при достижении скорости Ландау. Однако при увеличении $L_{2}$ резонанс между текучим и неметастабильным состояниями наступает при скоростях, меньших скорости Ландау, и это объясняет зависимость критической скорости от толшины капилляра.

Рассмотрим теперь состояние с наименьшей энергией из всех состояний, для которых $k_{1} \neq 0$ и $k_{2} \neq 0$. В таких состояниях существует как поперечная стоячая волна, так и продольное течение. Эти состояния также являются неметастабильными. Минимальную энергию имеет состояние, в котором $k_{2}=2 \pi\left(0,1 / L_{2}, 0\right)$ и $k_{1}=2 \pi\left(1 / L_{1}, 0,0\right)$, согласно формуле (78) эта энергия равна

$$
E_{\min }=N\left(\frac{\hbar^{2}(2 \pi)^{2}}{2 m}\left(\frac{1}{L_{1}^{2}}+\frac{1}{L_{2}^{2}}\right)+\frac{V_{0}}{2}\right)
$$

Соответствуюшая критическая скорость выражается формулой

$$
v_{\mathrm{c}}\left(L_{1}, L_{2}\right) \equiv \frac{2 \pi \hbar}{m} \sqrt{\frac{1}{L_{1}^{2}}+\frac{1}{L_{2}^{2}}}
$$

Благодарности. Автор приносит глубокую благодарность Г. В. Ковалю за помошь в счете.

\section{Список литературы}

[1] В. П. Маслов. ТМФ. 2002. Т. 132. № 3. С. 388-398.

[2] В. П. Маслов. Квантование термодинамики и ультравторичное квантование. М.: Институт компьютерных исследований, 2001.

[3] Ф. А. Березин. Метод вторичного квантования. М.: Наука, 1965.

[4] В. П. Маслов. Операторные методы. М.: Наука, 1973.

[5] В. П. Маслов, О. Ю. Шөедов. Метод комплексного ростка в задаче многих частиц и квантовой теории поля. М.: Эдиториал УРСС, 2000.

[6] Н. Н. Боголюбов. Избранные труды в трех томах. Т. 2. Киев: Наукова думка, 1970.

[7] М. В. Федорюк. УМН. 1971. Т. 26. № 1. С. 67-112.

[8] В. П. Маслов. Комплексный метод ВКБ в нелинейных уравнениях. М.: Наука, 1977. 